University of South Carolina

Scholar Commons

2-8-1999

\title{
Cumulative Reaction Probability in Terms of Reactant-Product Wave Packet Correlation Functions
}

Sophya Garashchuk

University of South Carolina--Columbia, sgarashc@chem.sc.edu

David J. Tannor

Follow this and additional works at: https://scholarcommons.sc.edu/chem_facpub

Part of the Chemistry Commons

Publication Info

Published in Journal of Chemical Physics, Volume 110, Issue 6, 1999, pages 2761-2770.

http://jcp.aip.org/

(c) 1999 by American Institute of Physics

This Article is brought to you by the Chemistry and Biochemistry, Department of at Scholar Commons. It has been accepted for inclusion in Faculty Publications by an authorized administrator of Scholar Commons. For more information, please contact digres@mailbox.sc.edu. 


\title{
Cumulative reaction probability in terms of reactant-product wave packet correlation functions
}

\author{
Sophya Garashchuk ${ }^{\text {a) }}$ \\ Department of Physics, Department of Chemistry \& Biochemistry, University of Notre Dame, Notre Dame, \\ Indiana 46556
}

David J. Tannor

Department of Chemical Physics, Weizmann Institute of Science, Rehovot, 76100, Israel

(Received 15 June 1998; accepted 6 November 1998)

\begin{abstract}
We present new expressions for the cumulative reaction probability $(N(E))$, cast in terms of time-correlation functions of reactant and product wave packets. The derivation begins with a standard trace expression for the cumulative reaction probability, expressed in terms of the reactive scattering matrix elements in an asymptotic internal basis. By combining the property of invariance of the trace with a wave packet correlation function formulation of reactive scattering, we obtain an expression for $N(E)$ in terms of the correlation matrices of incoming and outgoing wave packets which are arbitrary in the internal coordinates. This formulation, like other recent formulations of $N(E)$, allows calculation of the quantum dynamics just in the interaction region of the potential, and removes the need for knowledge of the asymptotic eigenstates. However, unlike earlier formulations, the present formulation is fully compatible with both exact and approximate methods of wave packet propagation. We illustrate this by calculating $N(E)$ for the collinear hydrogen exchange reaction, both quantally and semiclassically. These results indicate that the use of wave packet cross-correlation functions, as opposed to a coordinate basis and flux operators, regularizes the semiclassical calculation, suggesting that the semiclassical implementation described here may be applied fruitfully to systems with more degrees of freedom. (C) 1999 American Institute of Physics. [S0021-9606(99)01706-7]
\end{abstract}

\section{INTRODUCTION}

Calculating probabilities of chemical reactions is one of the central goals of theoretical chemistry. The complete information about a scattering process is contained in the scattering matrix, $S$, defined through the overlap of scattering eigenstates referred to different sets of boundary conditions $^{1,2}$

$$
S_{\beta \alpha}(E) \delta\left(E-E^{\prime}\right)=\left\langle\psi_{\beta, E}^{-} \mid \psi_{\alpha, E^{\prime}}^{+}\right\rangle .
$$

An individual matrix element gives the probability amplitude of a transition from the internal state $\alpha$ of reactants to the internal state $\beta$ of products at total energy $E,\left|S_{\beta \alpha}(E)\right|^{2}$. However, for many chemical reactions at energies of interest there may be hundreds or thousands of energetically accessible internal states and one may be interested only in the averaged quantity, the cumulative reaction probability $(N(E))$, defined as

$$
N(E)=\sum_{\alpha \beta}\left|S_{\beta \alpha}(E)\right|^{2}
$$

The Boltzmann average of $N(E)$ is, within a normalization factor, the thermal reaction rate constant $k(T)$, which is perhaps the most important single quantity for characterizing a chemical reaction.

${ }^{a)}$ Electronic mail: sgarashc@ rainbow.uchicago.edu
One can use the straightforward definition of Eq. (2) to find $N(E)$, but it is more efficient numerically and appealing conceptually to find $N(E)$ using alternative expressions which make no explicit reference to the asymptotic eigenstates. One of the most elegant expressions of this type is that of Miller, Schwartz, and Tromp ${ }^{3}$

$$
N(E)=\frac{(2 \pi \hbar)^{2}}{2} \operatorname{Tr}(\bar{F} \delta(E-H) \bar{F} \delta(E-H)),
$$

where $H$ is the Hamiltonian of a system and $\bar{F}$ is the symmetrized flux operator. Equation (3) has two main advantages: It can be evaluated in any complete basis and it allows one to consider dynamics in the interaction region only. An alternative formulation, due to Manthe, Seideman, and Miller, ${ }^{4-6}$ expresses $N(E)$ as

$$
N(E)=\operatorname{Tr}\left(4 \hat{\epsilon}_{r}^{1 / 2} \hat{G}(E)^{\dagger} \hat{\epsilon}_{p} \hat{G}(E) \hat{\epsilon}_{r}^{1 / 2}\right) .
$$

The operators $\hat{\epsilon}_{r, p}$ are asymptotic absorbing potentials which impose the outgoing boundary condition on the Green function $\hat{G}(E)$. This approach has all the advantages of Eq. (3) but appears to be more efficient numerically than the fluxbased approach.

Recently, Light and Zhang presented a time-dependent version of Eq. (3), which they call the quantum transition state wave packet method. ${ }^{7-10}$ The trace is evaluated in the basis constructed as eigenfunctions of the flux operator, 
$\bar{F}|+\rangle=\lambda|+\rangle$, and a set of internal functions of a transition state or any surface separating reactants and products, $\phi_{i}$

$$
N(E)=\sum_{i}\left\langle\psi_{i}|\bar{F}| \psi_{i}\right\rangle
$$

The energy wave functions $\psi_{i}$ are the Fourier transforms of the time-dependent wave functions

$$
\left.\left|\psi_{i}\right\rangle=\sqrt{\lambda} \int_{-\infty}^{\infty} e^{\imath(E-H) t}\left(|+\rangle \times \phi_{i}\right\rangle\right) d t .
$$

Another modification of Eq. (3) uses the spectral representation of $\hat{F}$, as in Eq. (4), for both flux operators. ${ }^{11}$

Despite the considerable progress in formulations of $N(E)$, full quantum-mechanical calculations of $N(E)$ are still limited to systems with no more than four atoms. The two main approaches to quantum dynamical calculations for high dimensional systems are semiclassical methods and mean field of time-dependent self-consistent field (TDSCF) methods, but there was little attention paid, if any, to formulating $N(E)$ in a way which can be conveniently implemented using these approximate techniques.

About five years ago, Tannor and Weeks presented a new formulation of state-to-state reactive scattering in which the individual $S$-matrix elements are expressed as Fourier transforms of a time cross-correlation function between reactant and product wave packets with specified asymptotic boundary conditions ${ }^{12-16}$ (for a related formulation, see Refs. 17 and 18). The central dynamical quantity in the method is an overlap between square normalizable states and thus the method is convenient for dynamical approximations; indeed, the approach has been combined successfully both with semiclassical ${ }^{19-21}$ and time-dependent self-consistent field $(\mathrm{TDSCF})^{22}$ propagation methods. Clearly, one could use the Tannor-Weeks formula to calculate $N(E)$ by summing over all initial and final states; however, the requirement to know the form of the asymptotic internal states, and the large number of energetically accessible asymptotic states in more complex systems, presents a severe impediment.

In this paper we present a new formulation of $N(E)$ which is in the spirit of the Tannor-Weeks formulation, but removes the requirement to know the asymptotic states or to propagate in from or out to the asymptotic region. The derivation starts with the definition of $N(E)$ in terms of the $S$-matrix elements between asymptotic internal eigenstates, Eq. (2), but utilizes the trace property of the cumulative reaction probability

$$
N(E)=\operatorname{Tr}\left(\boldsymbol{S}_{p r}^{\dagger} \boldsymbol{S}_{p r}\right),
$$

to express $N(E)$ in terms of wave packet correlation matrices in an arbitrary basis of internal coordinates. The wave packets are required to be incoming and outgoing in the translational degree of freedom but do not have to be started in the asymptotic region, so long as they are set up in the "nonreflecting" region of the potential. In the internal coordinates, the wave functions must form a complete set, but are otherwise arbitrary and need not be normalized or orthogonal.

The present formulation, like the work of Light and Zhang, is implemented in terms of wave packets, but like the work of Manthe and Miller avoids the use of flux operators. Expressing $N(E)$ in terms of wave packet correlation functions seems to regularize the problem, making the method well suited for both exact and approximate propagation methods. We illustrate this by the semiclassical calculation of $N(E)$ for the collinear hydrogen exchange reaction. The semiclassical calculation of $N(E)$ to date has been limited to a single study on a one-dimensional system, ${ }^{23}$ and thus the present semiclassical calculation of collinear hydrogen exchange is a strong indication of the utility of the new formulation. In fact, we see no reason that the present approach with its semiclassical implementation should not be applicable, in principle, to larger systems of greater chemical interest.

The paper is organized as follows. Section II provides a simplified derivation of the wave packet correlation formulation for individual $S$-matrix elements. This is important background, since correlation functions of this type are the basic building blocks for the new expressions for $N(E)$ in Sec. III and since the new expression for $N(E)$ reduces to a sum over squares of these expressions for the individual $S$-matrix elements in the special case that the wave packet basis uses the asymptotic internal eigenstates. Section III provides the derivation of the new formula. In this section we also present another expression, that uses correlation functions of wave packets projected onto the product (or reactant) asymptotic region, which is a generalization of a recent wave packet correlation function formulation for initialstate-selected total reaction probabilities ${ }^{24}$ to initial states in an arbitrary internal basis. Section IV illustrates the method by calculating $N(E)$ for the collinear hydrogen exchange reaction, using both quantum-mechanical propagation and the semiclassical propagator of Herman and Kluk. Section V concludes.

\section{SCATTERING MATRIX FROM WAVE PACKET TIME-CORRELATION FUNCTIONS}

\section{A. Preliminaries}

Consider the scattering of a reactive system with internal degrees of freedom. A Hamiltonian $H(R, r)$ governs the dynamics of the system, where $R$ is a translational coordinate and $r$ is a set of internal coordinates. By assumption, the interaction between fragments vanishes when the fragments are far apart. This means that $H$ can be written as a sum of an asymptotic Hamiltonian, $H_{\alpha}^{0}\left(H_{\beta}^{0}\right)$, and an interaction potential, $V_{\alpha}\left(V_{\beta}\right),\left(H=H_{\alpha}^{0}+V_{\alpha}=H_{\beta}^{0}+V_{\beta}\right)$ where $V_{\alpha}\left(V_{\beta}\right) \rightarrow 0$ for $R \rightarrow \infty$. The energy eigenfunctions of $H$ may be labeled as incoming $\left(^{+}\right)$or outgoing $\left({ }^{-}\right)$, depending on the direction of the translational wave relative to the interaction region

$$
H\left|\psi_{\alpha, E}^{+}\right\rangle=E\left|\psi_{\alpha, E}^{+}\right\rangle \quad \text { and } H\left|\psi_{\beta, E}^{-}\right\rangle=E\left|\psi_{\beta, E}^{-}\right\rangle .
$$

The first set of energy eigenstates $\left\{\psi_{\alpha, E}^{+}\right\}$correlates, i.e., is equivalent in the asymptotic region, to the eigenstates of $H_{\alpha}^{0}$ of the same energy $E$, with the same internal label $\alpha$ and incoming towards the interaction region from the reactant side. The second set $\left\{\psi_{\beta, E}^{-}\right\}$correlates with the eigenstate of 
$H_{\beta}^{0}$ of the same energy $E$ and with the same internal label $\beta$, but moving out of the interaction region on the product side. The parameter $E$ is the total energy of the system. All energy eigenfunctions within both sets are orthogonal to each other

$$
\left\langle\psi_{\alpha, E}^{+} \mid \psi_{\alpha^{\prime}, E^{\prime}}^{+}\right\rangle=\delta_{\alpha \alpha^{\prime}} \delta\left(E-E^{\prime}\right)
$$

and

$$
\left\langle\psi_{\beta, E}^{-} \mid \psi_{\beta^{\prime}, E^{\prime}}^{-}\right\rangle=\delta_{\beta \beta^{\prime}} \delta\left(E-E^{\prime}\right) .
$$

The relation between the two sets is expressed through matrix elements

$$
\left\langle\psi_{\beta, E^{\prime}}^{-} \mid \psi_{\alpha, E}^{+}\right\rangle=S_{\beta \alpha}(E) \delta\left(E-E^{\prime}\right),
$$

that form the $S$-matrix $S(E)=\left\{S_{\beta \alpha}(E)\right\}$.

\section{B. Expression for the S-matrix elements}

In the time-dependent framework that we exploit here, the fragments are described as wave packets localized in space and spread in energy:

$$
\left|\Phi_{\alpha}^{+}\right\rangle=\int_{0}^{\infty} \eta_{\alpha}(E)\left|\psi_{\alpha, E}^{+}\right\rangle d E
$$

and

$$
\left|\Phi_{\beta}^{-}\right\rangle=\int_{0}^{\infty} \zeta_{\beta}(E)\left|\psi_{\beta, E}^{-}\right\rangle d E .
$$

In the infinite past the reactant wave packet $\left|\Phi_{\alpha}^{+}\right\rangle$is located in the asymptotic region of the reactants. We require $\left|\Phi_{\alpha}^{+}\right\rangle$to be a direct product of an incoming wave packet in the translational degree of freedom and an eigenstate of the internal reactant Hamiltonian with quantum numbers $\alpha$ in the infinite past. We can construct such a wave packet, since the Hamiltonian is separable in the internal and translational degrees of freedom in the asymptotic region. The overall direction of motion of $\left|\Phi_{\alpha}^{+}\right\rangle$in the translational coordinate is towards the interaction region. Similarly, the product wave packet $\left|\Phi_{\beta}^{-}\right\rangle$ has to be in the asymptotic region of products in the infinite future. The translational motion is separated from the internal degrees of freedom, described by a single set of internal quantum numbers $\beta$. This wave packet is purely outgoing, i.e., it moves away from the interaction region when propagated forward in time under $H_{\beta}^{0}$. We use $\hbar=1$ throughout the paper. The Fourier transform of the time-correlation function of the reactant and product wave packets, $\left|\Phi_{\alpha}^{+}\right\rangle$and $\left|\Phi_{\beta}^{-}\right\rangle$, using Eqs. (6) and (8), gives the element $S_{\beta \alpha}(E)$ of the $S$-matrix

$$
\begin{array}{rl}
\int_{-\infty}^{\infty} d & t\left\langle\Phi_{\beta}^{-}\left|e^{-\imath H t}\right| \Phi_{\alpha}^{+}\right\rangle e^{\imath E t} \\
= & \int_{-\infty}^{\infty} d t\left(\int_{0}^{\infty} d E^{\prime} \zeta_{\beta}^{*}\left(E^{\prime}\right)\left\langle\psi_{\beta, E^{\prime}}^{-}\right|\right) e^{-\imath H t} \\
& \times\left(\int_{0}^{\infty} d E^{\prime \prime} \eta_{\alpha}\left(E^{\prime \prime}\right)\left|\psi_{\alpha, E^{\prime \prime}}^{+}\right\rangle\right) e^{\imath E t} \\
= & 2 \pi \int_{0}^{\infty} \int_{0}^{\infty} d E^{\prime} d E^{\prime \prime} \delta\left(E-E^{\prime \prime}\right) \zeta_{\beta}^{*}\left(E^{\prime}\right) \eta_{\alpha}\left(E^{\prime \prime}\right)
\end{array}
$$

$$
\begin{aligned}
& \times\left\langle\psi_{\beta^{\prime}, E^{\prime}}^{-} \mid \psi_{\alpha, E^{\prime \prime}}^{+}\right\rangle \\
= & 2 \pi \int_{0}^{\infty} d E^{\prime} \zeta_{\beta}^{*}\left(E^{\prime}\right) \eta_{\alpha}(E)\left\langle\psi_{\beta, E^{\prime}}^{+} \mid \psi_{\alpha, E}^{+}\right\rangle \\
= & 2 \pi \int_{0}^{\infty} d E^{\prime} \zeta_{\beta}^{*}\left(E^{\prime}\right) \eta_{\alpha}(E) \delta\left(E-E^{\prime}\right) S_{\beta \alpha}(E) \\
= & 2 \pi \zeta_{\beta}^{*}(E) \eta_{\alpha}(E) S_{\beta \alpha}(E) .
\end{aligned}
$$

Thus, we obtain the expression of Tannor and Weeks for the $S$-matrix element

$$
S_{\beta \alpha}(E)=\frac{(2 \pi)^{-1}}{\zeta_{\beta}^{*}(E) \eta_{\alpha}(E)} \int_{-\infty}^{\infty}\left\langle\Phi_{\beta}^{-}\left|e^{-\imath H t}\right| \Phi_{\alpha}^{+}\right\rangle e^{\imath E t} d t .
$$

The energy expansion coefficients $\eta_{\alpha}(E)$ and $\zeta_{\beta}(E)$ can be found analytically as an overlap of a wave packet and an energy eigenstate of the asymptotic Hamiltonian. ${ }^{12} \mathrm{We}$ found it convenient and essential for further derivation of $N(E)$ to use autocorrelation functions of wave packets to find the energy normalization functions $\left|\zeta_{\beta}(E)\right|$ and $\left|\eta_{\alpha}(E)\right|$. The Fourier transform of a time-correlation function of two reactant wave packets, using Eqs. (6) and (7), is

$$
\begin{aligned}
\int_{-\infty}^{\infty}\langle & \left.\Phi_{\alpha^{\prime}}^{+}\left|e^{-\imath H t}\right| \Phi_{\alpha}^{+}\right\rangle e^{\imath E t} d t \\
= & \int_{-\infty}^{\infty} d t\left(\int_{0}^{\infty} d E^{\prime} \eta_{\alpha^{\prime}}^{*}\left(E^{\prime}\right)\left\langle\psi_{\alpha^{\prime}, E^{\prime}}^{+}\right|\right) e^{-\imath H t} \\
& \times\left(\int_{0}^{\infty} d E^{\prime \prime} \eta_{\alpha}\left(E^{\prime \prime}\right)\left|\psi_{\alpha, E^{\prime \prime}}^{+}\right\rangle\right) e^{\imath E t} \\
= & 2 \pi \int_{0}^{\infty} \int_{0}^{\infty} d E^{\prime} d E^{\prime \prime} \delta\left(E-E^{\prime \prime}\right) \eta_{\alpha^{\prime}}^{*}\left(E^{\prime}\right) \eta_{\alpha}\left(E^{\prime \prime}\right) \\
& \times\left\langle\psi_{\alpha^{\prime}, E^{\prime}}^{+} \mid \psi_{\alpha, E^{\prime \prime}}^{+}\right\rangle \\
= & 2 \pi \int_{0}^{\infty} d E^{\prime} \eta_{\alpha^{\prime}}^{*}\left(E^{\prime}\right) \eta_{\alpha}(E)\left\langle\psi_{\alpha^{\prime}, E^{\prime}}^{+} \mid \psi_{\alpha, E}^{+}\right\rangle \\
= & 2 \pi \int_{0}^{\infty} d E^{\prime} \eta_{\alpha^{\prime}}^{*}\left(E^{\prime}\right) \eta_{\alpha}(E) \delta\left(E-E^{\prime}\right) \delta_{\alpha \alpha^{\prime}} \\
= & 2 \pi \eta_{\alpha^{\prime}}^{*}(E) \eta_{\alpha}(E) \delta_{\alpha^{\prime} \alpha} .
\end{aligned}
$$

Similarly, for product wave packets

$$
\int_{-\infty}^{\infty}\left\langle\Phi_{\beta^{\prime}}^{-}\left|e^{-\imath H t}\right| \Phi_{\beta}^{-}\right\rangle e^{\imath E t} d t=2 \pi \zeta_{\beta^{\prime}}^{*}(E) \zeta_{\beta}(E) \delta_{\beta^{\prime} \beta} .
$$

From Eqs. (12) and (13) the energy normalization functions are

$$
\left|\eta_{\alpha}(E)\right|=\left|(2 \pi)^{-1} \int_{-\infty}^{\infty}\left\langle\Phi_{\alpha}^{+}\left|e^{-\imath H t}\right| \Phi_{\alpha}^{+}\right\rangle e^{\imath E t} d t\right|^{1 / 2}
$$

and

$$
\left|\zeta_{\beta}(E)\right|=\left|(2 \pi)^{-1} \int_{-\infty}^{\infty}\left\langle\Phi_{\beta}^{-}\left|e^{-\imath H t}\right| \Phi_{\beta}^{-}\right\rangle e^{\imath E t} d t\right|^{1 / 2} .
$$

Combining Eq. (11) and Eqs. (14) and (15) we obtain 


$$
\left|S_{\beta \alpha}(E)\right|^{2}=\frac{\left|\int_{-\infty}^{\infty}\left\langle\Phi_{\beta}^{-}\left|e^{-\imath H t}\right| \Phi_{\alpha}^{+}\right\rangle e^{\imath E t} d t\right|^{2}}{\left|\int_{-\infty}^{\infty}\left\langle\Phi_{\beta}^{-}\left|e^{-\imath H t}\right| \Phi_{\beta}^{-}\right\rangle e^{\imath E t} d t \int_{-\infty}^{\infty}\left\langle\Phi_{\alpha}^{+}\left|e^{-\imath H t}\right| \Phi_{\alpha}^{+}\right\rangle e^{\imath E t} d t\right|} .
$$

The phase of the energy coefficients is irrelevant, since the reaction probability is $\left|S_{\beta \alpha}(E)\right|^{2}$.

There are several points worth mentioning about the $S$-matrix expression, Eq. (11):

1. A single calculation gives a column of the $S$-matrix for a range of energies that depends on the choice of reactant and product wave packets.

2. Equation (11) is symmetric with respect to reactants and products, and explicitly time reversible.

3. We can propagate the wave packets, $\left|\Phi_{\alpha}^{+}\right\rangle$forward in time and $\left|\Phi_{\beta}^{-}\right\rangle$backwards in time, towards each other. This leads to a shorter propagation for each wave packet which leads, in turn, to more localized wave packets hence a smaller grid.

4. Note that no additional wave packet propagation is necessary to obtain the energy normalization factors, Eqs. (14) and (15), since they can be calculated at the same time as the cross-correlation function in the numerator.

To implement Eq. (11) we have to find internal eigenstates of the asymptotic Hamiltonians $H_{\alpha}^{0}$ and $H_{\beta}^{0}$ and propagate wave packets starting in the asymptotic regions of fragments. We will show below that this is not necessary if we consider the cumulative reaction probability $N(E)$, since the reactive dynamics is determined entirely in the interaction region where rearrangement of fragments takes place. This conclusion is consistent with the trace formula for $N(E)$, Eq. (3). Finally, we note that the reaction probability, expressed as a ratio of the Fourier transforms of the wave packet correlation functions, Eq. (16), is suggestive of the expression for the cumulative reaction probability in the arbitrary internal state basis set, that will be derived in Sec. III A.

\section{NEW EXPRESSIONS FOR THE CUMULATIVE REACTION PROBABILITY}

\section{A. $N(E)$ based on the reactant/product wave packet time-correlation functions}

Consider a system with $N+1$ internal eigenstates in the asymptotic channels of products and reactants. Take two sets of wave packets, $\left\{\Phi_{r}\right\}$ on the reactant side of the potential and $\left\{\boldsymbol{\Phi}_{p}\right\}$ on the product side. These wave packets are some unknown linear combinations of the energy eigenstates with different internal quantum number, $\left\{\left|\psi_{n, E}^{+}\right\rangle\right\}$with incoming boundary conditions for reactant wave packets

$$
\left\{\begin{array}{l}
\left|\Phi_{r}^{0}\right\rangle=\int\left(\eta_{00}(E)\left|\psi_{0, E}^{+}\right\rangle+\cdots+\eta_{0 N}(E)\left|\psi_{N, E}^{+}\right\rangle\right) d E \\
\vdots \\
\left|\Phi_{r}^{N}\right\rangle=\int\left(\eta_{N 0}(E)\left|\psi_{0, E}^{+}\right\rangle+\cdots+\eta_{N N}(E)\left|\psi_{N, E}^{+}\right\rangle\right) d E
\end{array}\right.
$$

and $\left\{\left|\psi_{n, E}^{-}\right\rangle\right\}$outgoing boundary conditions for product wave packets

$$
\left\{\begin{array}{c}
\left|\Phi_{p}^{0}\right\rangle=\int\left(\zeta_{00}(E)\left|\psi_{0, E}^{-}\right\rangle+\cdots+\zeta_{0 N}(E)\left|\psi_{N, E}^{-}\right\rangle\right) d E \\
\vdots \\
\left|\Phi_{p}^{N}\right\rangle=\int\left(\zeta_{N 0}(E)\left|\psi_{0, E}^{-}\right\rangle+\cdots+\zeta_{N N}(E)\left|\psi_{N, E}^{-}\right\rangle\right) d E
\end{array}\right.
$$

The energy expansion coefficients can be arranged into matrices, where the first index $i=0 \cdots N$ labels wave packets and the second index $\alpha / \beta=0 \cdots N$ labels energy eigenfunctions, that are functions of the energy

$$
\boldsymbol{M}_{r}(E)=\left\{\eta_{i \alpha}(E)\right\}
$$

and

$$
\boldsymbol{M}_{p}(E)=\left\{\zeta_{i \beta}(E)\right\} .
$$

Thus, Eqs. (17) and (18) can be rewritten as

$$
\left(\left|\Phi_{r}^{0}\right\rangle \cdots\left|\Phi_{r}^{N}\right\rangle\right)=\int_{0}^{\infty} d E\left(\left|\psi_{0, E}^{+}\right\rangle \cdots\left|\psi_{N, E}^{+}\right\rangle\right) \boldsymbol{M}_{r}(E)
$$

for reactants, and

$$
\left(\left|\Phi_{p}^{0}\right\rangle \cdots\left|\Phi_{p}^{N}\right\rangle\right)=\int_{0}^{\infty} d E\left(\left|\psi_{0, E}^{-}\right\rangle \cdots\left|\psi_{N, E}^{-}\right\rangle\right) \boldsymbol{M}_{p}(E)
$$

for products. We emphasize that the matrices, $\boldsymbol{M}_{r / p}$, are unknown, and will not appear in our final working expression. Here and below we decided to write down vectors explicitly to avoid additional indices which the tensor notations would require.

Now we propagate the reactant wave packets and calculate all the correlation functions among themselves

$$
C_{r}^{i j}(t)=\left\langle\Phi_{r}^{i}\left|\exp \left(-{ }_{\imath} H t\right)\right| \Phi_{r}^{j}\right\rangle, \quad\{i, j\}=0 \cdots N
$$

This defines an $(N+1) \times(N+1)$ matrix. We may Fourier transform each of the elements of this matrix to obtain

$$
A_{r}^{i j}(E)=\int_{-\infty}^{\infty} d t C_{r}^{i j}(t) e^{i E t}
$$

The numbers define a new matrix, $\boldsymbol{A}_{r}(E)$. Substituting Eqs. (21) and (23) into Eq. (24), and using Eq. (12), it is readily verified that $\boldsymbol{A}_{r}(E)$ may be written as 


$$
\begin{aligned}
\boldsymbol{A}_{r}(E)= & \int_{-\infty}^{\infty} d t \int_{0}^{\infty} d E^{\prime} \boldsymbol{M}_{r}^{\dagger}\left(E^{\prime}\right)\left(\begin{array}{c}
\left\langle\psi_{0, E^{\prime}}^{+}\right| \\
\vdots \\
\left\langle\psi_{N, E^{\prime}}^{+}\right|
\end{array}\right) \\
& \times e^{-\imath H t} \int_{0}^{\infty} d E^{\prime \prime}\left(\left|\psi_{0, E^{\prime \prime}}^{+}\right\rangle \cdots\left|\psi_{N, E^{\prime \prime}}^{+}\right\rangle\right) \boldsymbol{M}_{r}\left(E^{\prime \prime}\right) e^{\imath E t} \\
= & 2 \pi \int_{0}^{\infty} d E^{\prime} \int_{0}^{\infty} d E^{\prime \prime} \boldsymbol{M}_{r}^{\dagger}\left(E^{\prime}\right) \\
& \times \delta\left(E^{\prime}-E^{\prime \prime}\right) \delta\left(E-E^{\prime \prime}\right) \boldsymbol{M}_{r}\left(E^{\prime \prime}\right) \\
= & 2 \pi \boldsymbol{M}_{r}^{\dagger}(E) \boldsymbol{M}_{r}(E),
\end{aligned}
$$

where $\delta\left(E^{\prime}-E^{\prime \prime}\right)$ comes from the orthogonality of energy eigenstates at different energies and $\delta\left(E-E^{\prime \prime}\right)$ comes from the time integral. Similarly, we define the product-product correlation functions $C_{p}^{i j}(t)=\left\langle\Phi_{p}^{i}|\exp (-\imath H t)| \Phi_{p}^{j}\right\rangle$, and its Fourier transform, $A_{p}^{i j}(E)$. Following a derivation analogous to that for reactants, the Fourier transforms of the productproduct correlation functions may be written in matrix form as:

$$
\boldsymbol{A}_{p}(E)=2 \pi \boldsymbol{M}_{p}^{\dagger}(E) \boldsymbol{M}_{p}(E) .
$$

Equations (25) and (26) provide a strategy for eliminating the unknown coefficients, $\boldsymbol{M}_{r}(E), \boldsymbol{M}_{p}(E)$ in terms of the known matrices $\boldsymbol{A}_{r}(E)$ and $\boldsymbol{A}_{p}(E)$, provided that the matrix $\boldsymbol{M}_{r / p}$ and its adjoint always come together; below we will show that this is indeed the case.

Finally, we define the reactant-product correlation functions $C_{i j}^{p r}(t)=\left\langle\Phi_{i}^{p}|\exp (-\imath H t)| \Phi_{j}^{r}\right\rangle$ for $\{i, j\}=0 \cdots N$. The Fourier transform of each of these reactant-product correlation functions can be written as $A_{p r}^{i j}(E)$. Using Eq. (10), the matrix of Fourier transformation of all the reactant-product correlation functions is readily seen to be

$$
\begin{aligned}
\boldsymbol{A}_{p r}(E)= & 2 \pi \int_{0}^{\infty} d E^{\prime} \boldsymbol{M}_{p}^{\dagger}\left(E^{\prime}\right)\left(\begin{array}{c}
\left\langle\psi_{0, E^{\prime}}^{-}\right| \\
\vdots \\
\left\langle\psi_{N, E^{\prime}}^{-}\right.
\end{array}\right) \\
& \times\left(\left|\psi_{0, E}^{+}\right\rangle \ldots\left|\psi_{N, E}^{+}\right\rangle\right) \boldsymbol{M}_{r}(E) \\
= & 2 \pi \boldsymbol{M}_{p}^{\dagger}(E) \boldsymbol{S}_{p r}(E) \boldsymbol{M}_{r}(E),
\end{aligned}
$$

where $\boldsymbol{S}_{p r}(E)$ is by definition the scattering matrix. More precisely, $\boldsymbol{S}_{p r}(E)$ is a part of the scattering matrix that describes chosen reactants and products. Note, that the product of the state vectors in Eq. (27) produces the matrix $S_{p r}(E)$ of dimension $(N+1)^{2}$. Formally, if there exists an inverse of the matrices $\boldsymbol{M}_{r}$ and $\boldsymbol{M}_{p}$, the $\boldsymbol{S}$-matrix may be written as

$$
\boldsymbol{S}_{p r}(E)=(2 \pi)^{-1} \boldsymbol{M}_{p}^{\dagger-1}(E) \boldsymbol{A}_{p r}(E) \boldsymbol{M}_{r}^{-1}(E) .
$$

Now, realizing that the cumulative reaction probability can be expressed as a trace

$$
N(E)=\operatorname{Tr}\left(S_{p r}(E) S_{p r}^{\dagger}(E)\right),
$$

and using Eq. (28) for the $S$-matrix, we can write

$$
\begin{aligned}
N(E)= & \operatorname{Tr}\left(\boldsymbol{M}_{p}^{\dagger-1}(E) \boldsymbol{A}_{p r}(E) \boldsymbol{M}_{r}^{-1}(E)\right. \\
& \left.\times \boldsymbol{M}_{r}^{\dagger-1}(E) \boldsymbol{A}_{p r}^{\dagger}(E) \boldsymbol{M}_{p}^{-1}(E)\right),
\end{aligned}
$$

Permutating matrices inside the trace and using Eqs. (25) and (26) we obtain

$$
N(E)=\operatorname{Tr}\left(\boldsymbol{A}_{p r}(E) \boldsymbol{A}_{r}^{-1}(E) \boldsymbol{A}_{p r}^{\dagger}(E) \boldsymbol{A}_{p}^{-1}(E)\right) .
$$

This is our final expression for $N(E)$ in terms of the dynamics of incoming and outgoing wave packets in an arbitrary basis of internal states. Note that all dependence on the matrices $\boldsymbol{M}_{r}$ and $\boldsymbol{M}_{p}$, which would require knowledge of the asymptotic states, has disappeared.

If the wave packets are initially located in the asymptotic region of the potential and they each correspond to a single internal eigenstate $\alpha$ and $\beta$, then Eq. (31) reduces to $N(E)$ $=\Sigma_{\alpha \beta}\left|S_{\beta \alpha}(E)\right|^{2}$. Each $S$-matrix element in this sum takes the form of Eq. (16).

\section{B. $N(E)$ based on the time-correlation of the wave packets and the projection operator for products}

In a previous paper ${ }^{24}$ we derived an expression for the total initial-state selected reaction probability $N_{\alpha}(E)$ $=\Sigma_{\beta}\left|S_{\beta \alpha}(E)\right|^{2}$ involving a projection onto products

$$
N_{\alpha}(E)=\frac{\int_{-\infty}^{\infty}\left\langle\Phi_{\alpha}^{+}\left|e^{-\imath H t}\right| \hat{P}_{p} \Phi_{\alpha}^{+}\right\rangle e^{\imath E t} d t}{\int_{-\infty}^{\infty}\left\langle\Phi_{\alpha}^{+}\left|e^{-\imath H t}\right| \Phi_{\alpha}^{+}\right\rangle e^{l E t} d t} .
$$

Here $\Phi_{\alpha}^{+}$is a purely incoming wave packet in the translational coordinate and $\alpha$ is an eigenstate of the internal coordinate in the asymptotic reactant region of the potential. The projection operator is

$$
\hat{P}_{p}=\lim _{t \rightarrow \infty} e^{\imath H t} h(s) e^{-\imath H t}, \quad \hat{P}_{p}^{2}=\hat{P}_{p},
$$

with $h(s)$ being the Heaviside function of the reaction coordinate $s$. A wave packet $\Phi_{\alpha}^{+}$is propagated forward in time until its bifurcation is complete. The nonreactive part of it is discarded, and the reactive part of the wave packet is propagated backwards in time. The autocorrelation function of the projected wave packet or, equivalently, the correlation function of the projected wave packet with the initial wave packet, is Fourier transformed into energy space and normalized to yield $N_{\alpha}(E)$. We now show that this expression also can be generalized for calculation of $N(E)$ in an arbitrary internal coordinate basis.

As in Sec. III A, we take a set of $N+1$ incoming wave packets, $\left\{\boldsymbol{\Phi}_{r}\right\}$ to span a space of $N+1$ internal states, as in Eq. (17). The matrix of Fourier transforms of all timedependent correlation functions that normalizes the energy eigenstates, is given by Eq. (25), i.e., $\boldsymbol{A}_{r}(E)$ $=2 \pi \boldsymbol{M}_{r}^{\dagger}(E) \boldsymbol{M}_{r}(E)$. As before, we assume that $\boldsymbol{M}_{r}$ is unknown but that $\boldsymbol{A}_{r}$ is readily computed. We define the matrix of Fourier transforms of the correlation functions of the projected wave packets with the initial wave packets as 


$$
A_{\text {proj }}(E)=\int_{0}^{\infty}\left(\begin{array}{c}
\left\langle\Phi_{r}^{0}\right| \\
\vdots \\
\left\langle\Phi_{r}^{N}\right|
\end{array}\right) e^{-i H t} \hat{P}_{p}\left(\left|\Phi_{r}^{0}\right\rangle \cdots\left|\Phi_{r}^{N}\right\rangle\right) e^{i E t} d t
$$

where

$$
\begin{aligned}
\hat{P}_{p} & =\sum_{\beta} \int_{0}^{\infty} d E^{\prime}\left|\psi_{\beta, E^{\prime}}^{-}\right\rangle\left\langle\psi_{\beta, E^{\prime}}^{-}\right| \\
& =\int_{0}^{\infty} d E^{\prime}\left(\left|\psi_{0, E^{\prime}}^{-}\right\rangle \cdots\left|\psi_{N, E^{\prime}}^{-}\right\rangle\right)\left(\begin{array}{c}
\left\langle\psi_{0, E^{\prime}}^{-}\right| \\
\vdots \\
\left\langle\psi_{0, E^{\prime}}^{-}\right|
\end{array}\right) .
\end{aligned}
$$

Note that Eq. (34) contains a product of a column with a row, generating a matrix.

The projection operator, Eq. (35), acts on the reactant wave packets, Eq. (19), yielding

$$
\begin{aligned}
\hat{P}_{p}\left(\left|\Phi_{r}^{0}\right\rangle \cdots\left|\Phi_{r}^{N}\right\rangle\right) \\
=\int_{0}^{\infty} d E^{\prime}\left(\left|\psi_{0, E^{\prime}}^{-}\right\rangle \cdots\left|\psi_{N, E^{\prime}}^{-}\right\rangle\right) \\
\quad \times\left(\begin{array}{c}
\left\langle\psi_{0, E^{\prime}}^{-}\right| \\
\vdots \\
\left\langle\psi_{0, E^{\prime}}^{-}\right|
\end{array}\right) \int_{0}^{\infty} d E\left(\left|\psi_{0, E}^{+}\right\rangle \cdots\left|\psi_{N, E}^{+}\right\rangle\right) \boldsymbol{M}_{r}(E) \\
=\int_{0}^{\infty} d E^{\prime} \int_{0}^{\infty} d E\left(\left|\psi_{0, E^{\prime}}^{-}\right\rangle \cdots\left|\psi_{N, E^{\prime}}^{-}\right\rangle\right) \boldsymbol{S}_{p r}(E) \\
\quad \times \delta\left(E-E^{\prime}\right) \boldsymbol{M}_{r}(E) \\
=\int_{0}^{\infty} d E\left(\left|\psi_{0, E^{\prime}}^{-}\right\rangle\left|\psi_{N, E^{\prime}}^{-}\right\rangle\right) \boldsymbol{S}_{p r}(E) \boldsymbol{M}_{r}(E),
\end{aligned}
$$

where we have used the definition of the $S$-matrix, Eq. (8). Substituting this result into Eq. (34) we obtain

$$
\begin{aligned}
A_{\mathrm{proj}}(E)= & \int_{-\infty}^{\infty} d t e^{i E t} \int_{0}^{\infty} d E^{\prime} \boldsymbol{M}_{r}^{\dagger}\left(E^{\prime}\right)\left(\begin{array}{c}
\left\langle\psi_{0, E^{\prime}}^{+}\right| \\
\vdots \\
\left\langle\psi_{0, E^{\prime}}^{+}\right.
\end{array}\right) e^{-i H t} \\
& \left.\times \int_{0}^{\infty} d E^{\prime \prime}\left(\left|\psi_{0, E^{\prime \prime}}^{-}\right\rangle \cdot \psi_{N, E^{\prime \prime}}^{-}\right\rangle\right) \boldsymbol{S}_{p r}\left(E^{\prime \prime}\right) \boldsymbol{M}_{r}\left(E^{\prime \prime}\right) \\
= & \int_{0}^{\infty} d E^{\prime} \int_{0}^{\infty} d E^{\prime \prime} \boldsymbol{M}_{r}^{\dagger}\left(E^{\prime}\right) \boldsymbol{S}_{p r}^{\dagger}\left(E^{\prime}\right) \\
& \times \delta\left(E^{\prime}-E^{\prime \prime}\right) 2 \pi \delta\left(E-E^{\prime \prime}\right) \boldsymbol{S}_{p r}\left(E^{\prime \prime}\right) \boldsymbol{M}_{r}\left(E^{\prime \prime}\right) \\
= & 2 \pi \boldsymbol{M}_{r}^{\dagger}(E) \boldsymbol{S}_{p r}^{\dagger}(E) \boldsymbol{S}_{p r}(E) \boldsymbol{M}_{r}(E) .
\end{aligned}
$$

Above, the integral over time yielded $2 \pi \delta\left(E-E^{\prime \prime}\right)$, and the product of the column $\left\{\left\langle\psi_{\alpha, E^{\prime}}^{+}\right|\right\}$by the row $\left\{\left|\psi_{\beta, E^{\prime \prime}}^{-}\right\rangle\right\}$gave the matrix $S_{p r}^{\dagger}\left(E^{\prime}\right) \delta\left(E^{\prime}-E^{\prime \prime}\right)$ according to Eq. (8).

Inverting this expression, taking a trace and permutating matrices inside the trace we arrive at

$$
\begin{aligned}
N(E) & =\operatorname{Tr}\left(\boldsymbol{S}_{p r}^{\dagger}(E) \boldsymbol{S}_{p r}(E)\right) \\
& =\operatorname{Tr}\left(\boldsymbol{M}_{r}^{\dagger-1}(E) \boldsymbol{A}_{\mathrm{proj}}(E) \boldsymbol{M}_{r}^{-1}(E)\right) \\
& =\operatorname{Tr}\left(\boldsymbol{A}_{\text {proj }}(E) \boldsymbol{A}_{r}^{-1}(E)\right) .
\end{aligned}
$$

Thus, we can evaluate $N(E)$ by propagating a set of incoming, otherwise arbitrary, wave packets, projecting onto products, and then calculating correlation functions of these projected packets. Similar to Sec. III A, in the basis of asymptotic internal energy eigenstates the expression (38) reduces to $N(E)=\sum_{\alpha} N_{\alpha}(E)$ with each term being of the form Eq. (32).

\section{NUMERICAL IMPLEMENTATION AND DISCUSSION}

\section{A. General remarks}

In order to derive Eq. (11) for the $S_{\beta \alpha}(E)$ and Eqs. (31) and (38) for $N(E)$ we assumed that the reactant wave packets $\left\{\boldsymbol{\Phi}_{r}\right\}$ are purely incoming and that the product wave packets $\left\{\boldsymbol{\Phi}_{p}\right\}$ are purely outgoing. A rigorous way to set up such purely incoming or outgoing wave packets is to construct them in the reactant (product) asymptotic regions as a direct product of incoming (outgoing) wave packets in the translational degree of freedom and arbitrary internal basis functions. However, in practice, we can bring the wave packets significantly closer to the interaction region, as long as we ensure that they correlate with the incoming (outgoing) translational wave packets in the infinite past (infinite future).

Recently, it was noted ${ }^{22,25}$ that for the $S$-matrix expressions, the restriction on pure incoming or outgoing wave packets can be removed if the lower limit $t=-\infty$ in the time integral in Eq. (11) is replaced by $t=0$, provided the energy normalization can be determined by some other means. The energy normalization is the overlap of the reactant (product) wave packets with eigenstates of incoming (outgoing) character. This overlap is trivial to calculate, provided the reactant (product) wave packets are also of pure incoming (outgoing) character: Since there is no amplitude other than that of incoming character, the energy normalization obtained from the wave packet correlation functions are the correct overlap factors. However, if the wave packet is of mixed incoming-outgoing character, the incoming-outgoing components would have to be separated before the overlap could be calculated. This tends to negate any savings from bringing the wave packets closer in, and below we use purely incoming and outgoing wave packets exclusively.

If we set up wave packets $\left\{\boldsymbol{\Phi}_{r}\right\}$ and $\left\{\boldsymbol{\Phi}_{p}\right\}$ in the asymptotic regions of the potential and work in the basis of energy eigenfunctions, then the number of wave packets we need is $N+1$ for each arrangement channel. If the internal basis is different from the internal energy eigenfunctions, the number of wave packets can be expected to be somewhat larger than $N+1$. However, if the translational wave packets are positioned closer to the interaction region, and we use an efficient internal basis, for example, energy eigenfunctions of the surface perpendicular to the reaction coordinate of the center of a wave packet, we can expect the number of wave packets to be smaller than $N+1$. In order to invert the ma- 
TABLE I. Numerical parameters in atomic units; Absorbing potential $V_{a}=\kappa h\left(R-R_{a}\right) h\left(r-r_{a}\right)$.

\begin{tabular}{ccc}
\hline \hline Grid size $128 \times 128$ & Grid spacing $d r=0.11$ & Grid starting point $r_{\min }=0.5$ \\
Time step $d t=7.29$ & Number of time steps 1000 & \\
$\kappa=4.439 \times 10^{-3}$ & translational coordinate $R_{a}=R_{0}+2.0$ & vibrational coordinate $r_{a}=4.2$ \\
\hline \hline
\end{tabular}

trices $A_{p}(E)$ and $A_{r}(E)$ in practice we use singular value decomposition (SVD), since, in general, the number of the internal functions is larger than the number of the energy eigenstates for a specific value of energy.

Equation (31) is explicitly time reversible, and reactants and products enter on an equal footing. Since all the reactant wave packets, $\left\{\boldsymbol{\Phi}_{r}\right\}$, are incoming and all the product wave packets, $\left\{\boldsymbol{\Phi}_{p}\right\}$, are outgoing, the reactant-product correlation functions are zero for negative times. Reactant-reactant (and product-product) correlation functions for positive and negative times are related to each other

$$
C_{r(p)}^{i j}(-t)=C_{r(p)}^{j i} *(t),
$$

and they die off faster in time than reactant-product correlation functions. Also, we can split the time propagation between reactant and product wave packets as

$$
C_{p r}^{i j}(t)=\left\langle\Phi_{p}^{i}\left|e^{-\imath H t}\right| \Phi_{r}^{j}\right\rangle=\left\langle e^{\imath H t / 2} \Phi_{p}^{i} \mid e^{-\imath H t / 2} \Phi_{r}^{j}\right\rangle .
$$

Thus, half-propagation of reactants, $\left\{\boldsymbol{\Phi}_{r}\right\}$, just forward in time and half-propagation of products, $\left\{\boldsymbol{\Phi}_{p}\right\}$, just backwards in time is sufficient for calculation of all the matrices involved in the expression for $N(E)$, Eq. (31). Thus we do not have to propagate reactant (product) wave packets back (forward) in time, and we can use an absorbing potential efficiently.

Equation (38), containing as it does a projection operator, does not have the reactant-product symmetry. The absorbing potential cannot be placed close to the interaction region on the product side. Nevertheless, this asymmetry might be useful if the number of the product eigenstates is significantly greater than the number of the reactant eigenstates.

\section{B. Numerical example: Collinear $\mathrm{H}_{2}+\mathrm{H}$ scattering}

We applied the formalism of Sec. III A, Eq. (31), to the collinear hydrogen exchange reaction on the potential energy surface of Liu, Siegban, Truhlar, and Horowitz (LSTH). ${ }^{26-28}$ Reactant wave packets $\left\{\boldsymbol{\Phi}_{r}\right\}$ were set up in the Jacobi coordinates, $\{R, r\}$, as a direct product of the vibrational eigenstates and a Gaussian in the translational coordinate $R$

$$
\Phi_{r}^{n}(R, r)=e^{-\gamma\left(R-R_{0}\right)^{2}+\imath p_{0}\left(R-R_{0}\right)} \times \chi_{n}(r) .
$$

The wave packets $\left\{\boldsymbol{\Phi}_{p}\right\}$ were setup in the product Jacobi coordinates and have the same parameters as the reactant wave packets, except for the sign of the translational momentum to make them outgoing

$$
\Phi_{p}^{n}\left(R^{\prime}, r^{\prime}\right)=e^{-\alpha\left(R^{\prime}-R_{0}\right)^{2}-\imath p_{0}\left(R^{\prime}-R_{0}\right)} \times \chi_{n}\left(r^{\prime}\right) .
$$

The quantum-mechanical $(\mathrm{QM})$ time propagation of the wave packets was performed using the split operator method $^{29}$ on a grid in bond coordinates with an imaginary absorbing potential. The numerical details are given in Table
I. In order to obtain the cumulative reaction probability for energies below $2.15 \mathrm{eV}$ we propagated wave packets with the vibrational quantum numbers from $n=0$ to $n=3$. We chose $\chi_{n}(r)$ to be the $n$th energy eigenstates of either the Morse oscillator

$$
V_{m}(r)=0.1743(\exp (-1.04435(r-1.40083))-1)^{2},
$$

or the harmonic oscillator

$$
V_{h}(r)=0.1898(r-1.40083)^{2} \text {. }
$$

Figure 1 shows $N(E)$ for two sets of wave packets using the Morse oscillator eigenstates. The first set, $k=1$ in Table II, was initially located in the asymptotic region of the potential at $R_{0}^{(1)}=6.7$ a.u. The second set, $k=2$ in Table II was placed in the interaction region at $R_{0}^{(2)}=4.7$ a.u. Small eigenvalues $\lambda$ were discarded in the SVD. The cutoff was $\lambda_{\min } / \lambda_{\max }$ $=0.02$. The cumulative reaction probabilities for the two calculations agree with each other for the range of energy values with slight discrepancies around the higher resonances. We attribute the small discrepancies to the fact that the wave packets with high quantum numbers $n$ from the second set substantially penetrate into the interaction region of the potential. Thus, they have larger overlaps with the resonance states, which makes it difficult to obtain accurate spectral amplitudes. We believe, that the problem with resonances can be remedied by using a high resolution spectral method, like filter-diagonalization, instead of the Fourier

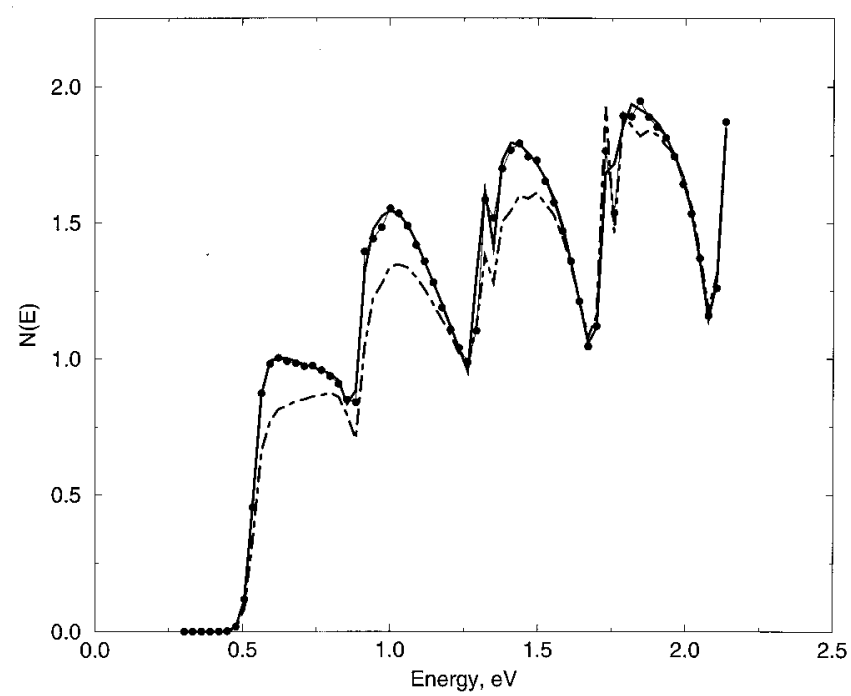

FIG. 1. The cumulative reaction probability calculated from the Eq. (31) using the Morse oscillator eigenstates as internal coordinate basis. The wave packets are located in translational coordinate in the asymptotic region at $R_{0}^{(1)}=6.7$ a.u. - circles, in the interaction region $R_{0}^{(2)}=4.7$ a.u. - solid line. Dashed line shows the results when the reactant-reactant correlation functions of the first, asymptotic region, set are used to normalize the reactant-product correlation functions of the second, interaction region, set of wave packets. 
TABLE II. Parameters of the initial wave packet $\Phi_{r}^{n}(R, r)$ $=e^{-\gamma\left(R-R_{0}\right)^{2}+\imath p_{0}\left(R-R_{0}\right)} \chi_{n}(r)$ in atomic units.

\begin{tabular}{cccccc}
\hline \hline $\mathrm{k}$ & 1 & 2 & 3 & 4 & 5 \\
$\chi_{n}(r)$ & Morse & Morse & Morse & Harmonic & Harmonic \\
$R_{0}^{(k)}$ & 6.7 & 4.7 & 4.2 & 4.7 & 4.2 \\
\hline for all $k$ & & $\gamma=6.0$ & $p_{0}=-8.0$ & $n=0.3$ & \\
\hline \hline
\end{tabular}

transform. ${ }^{30-32}$ This method works best when combined with the Chebyshev propagator to obtain very accurate timecorrelation functions, or with the Chebyshev recursion which can be used as an alternative to time correlation functions.

Figure 1 also shows that the energy normalization in the two calculations is, indeed, different. The long-dashed curve represents $N(E)$ obtained using wave packets that were placed in the interaction region, but the energy normalization derived from the asymptotic wave packet dynamics. In this case, the change in the normalization for the asymptotic and interaction region calculations is essentially a shift in energy, as seen in Fig. 2. The maximal amplitude of the off-diagonal reactant-reactant correlation functions $C_{r}^{i j}(i \neq j)$ was about 0.03 for the set (1) and 0.07 for the set (2) for wave functions normalized to unity. We also obtained $N(E)$ using two different sets of harmonic oscillator eigenstates, located at $R_{0}^{(4)}=4.7$ a.u. and $R_{0}^{(5)}=4.2$ a.u. $(k=4,5$ in Table II). The matrix eigenvalue cutoff was $\lambda_{\min } / \lambda_{\max }=0.03$. The harmonic eigenstates are tighter than the Morse eigenstates. They do not penetrate into the interaction region as much as the Morse eigenstates, yet they adequately describe the reaction for the chosen range of energy. The cumulative reaction probability for these wave packets is plotted in Fig. 3. Figure 4 compares $N(E)$ for the harmonic and Morse oscillator eigenstates ( $k=3$ in Table II) initially placed at $R_{0}=4.2$ a.u. One can see that, as a result of being more localized, the harmonic oscillator eigenstates give better results than the Morse eigenstates for near resonant energies.

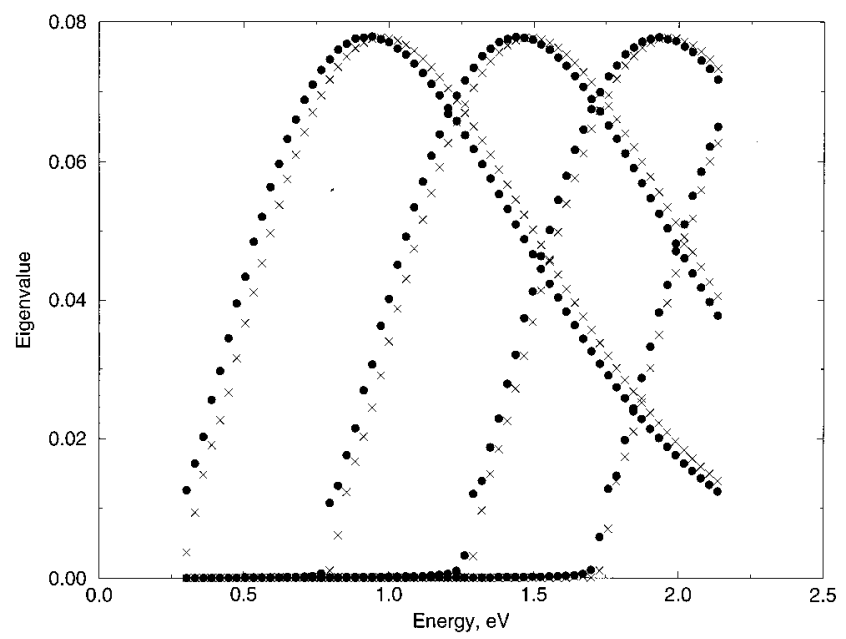

FIG. 2. The eigenvalues of the energy normalization matrices, $A_{r}(E)$ and $A_{p}(E)$, as a function of energy for the asymptotic wave packet set $\left(R_{0}^{(1)}\right.$ $=6.7$ a.u. $)-$ circles and the interaction region wave packet set $\left(R_{0}^{(2)}\right.$ $=4.7$ a.u. $)$ - crosses.

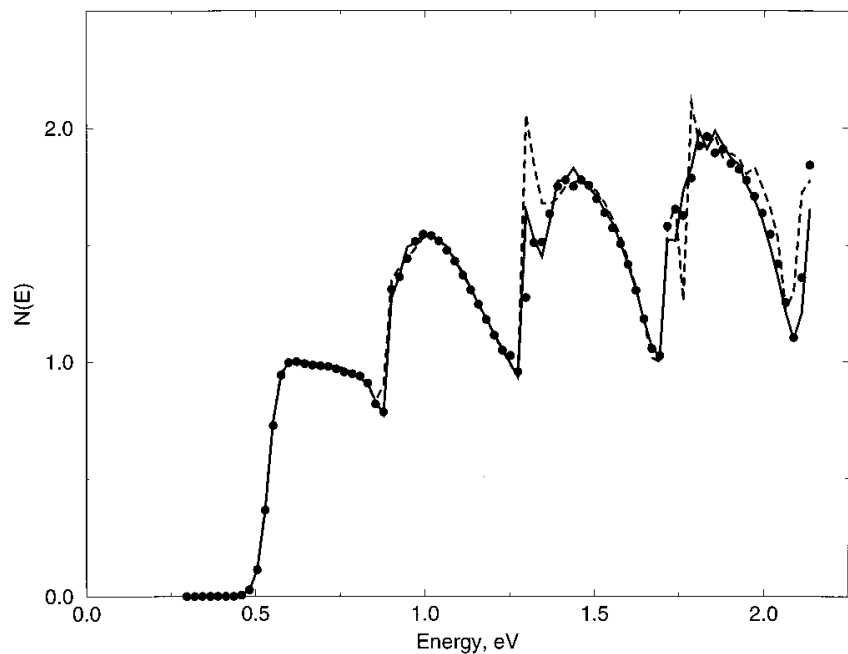

FIG. 3. The cumulative reaction probability calculated using the harmonic oscillator eigenstates as $\chi_{n}(r)$ and initially located $R_{0}^{(4)}=4.7$ a.u. - solid line; using the harmonic oscillator eigenstates and $R_{0}^{(5)}=4.2$ a.u. - dashed line, compared to the results of the wave packet set (1).

\section{Cumulative reaction probability for the collinear hydrogen exchange reaction: Semiclassical implementation}

We also implemented Eq. (31) using the semiclassical propagator of Herman and Kluk $(\mathrm{HK})^{33}$

$$
\begin{aligned}
K^{s c}\left(\boldsymbol{x}^{\prime}, t ; \boldsymbol{x}, 0\right)= & \frac{1}{(2 \pi \hbar)^{N}} \iint d \boldsymbol{p}_{0} d \boldsymbol{q}_{0} R_{p q t} e^{\iota S_{p q t} / \hbar} \\
& \times g_{\gamma}\left(\boldsymbol{q}_{t}, \boldsymbol{p}_{t}, \boldsymbol{x}^{\prime}\right) g_{\gamma}^{*}\left(\boldsymbol{q}_{0}, \boldsymbol{p}_{0}, \boldsymbol{x}\right) .
\end{aligned}
$$

A function

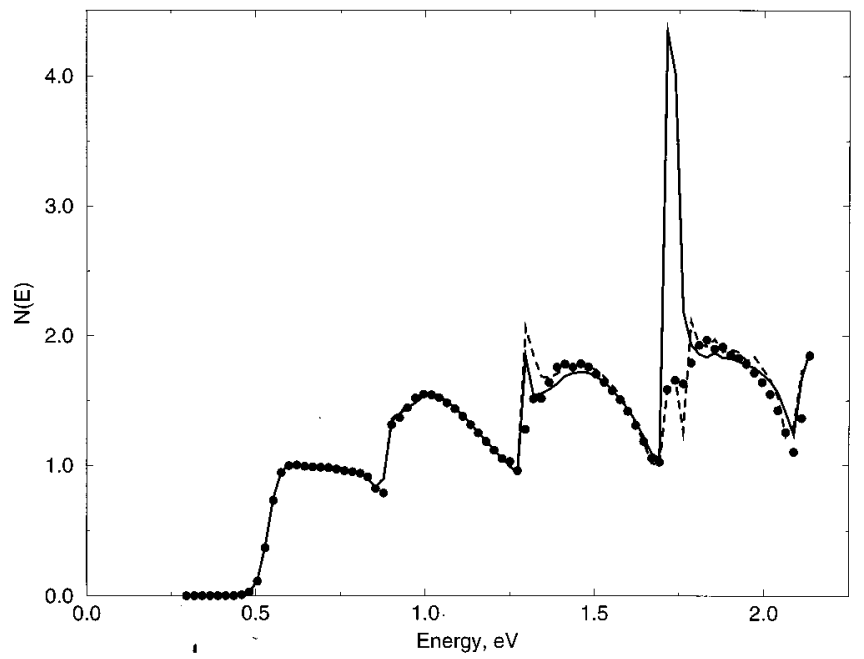

FIG. 4. $N(E)$ for the wave packets located at $R_{0}^{(3)}=4.2$ a.u. using Morse eigenstates (solid line) and located at $R_{0}^{(5)}=4.2$ a.u. (dash line) using harmonic eigenstates, compared to the result of the wave packet set (1) (circles). 


$$
\begin{aligned}
g_{\gamma}\left(\boldsymbol{q}_{t}, \boldsymbol{p}_{t}, \boldsymbol{x}\right)= & \left(\frac{\gamma}{\pi}\right)^{N / 4} \\
& \times \exp \left(-\frac{\gamma}{2}\left(\boldsymbol{x}-\boldsymbol{q}_{t}\right)\left(\boldsymbol{x}-\boldsymbol{q}_{t}\right)+\frac{l}{\hbar} \boldsymbol{p}_{t} \cdot\left(\boldsymbol{x}-\boldsymbol{q}_{t}\right)\right),
\end{aligned}
$$

is a complex Gaussian of the width $\gamma$, which is a positive real parameter. Vectors $\boldsymbol{q}_{0}=\left(q_{0}^{1}, \ldots, q_{0}^{N}\right)$ and $\boldsymbol{p}_{0}=\left(p_{0}^{1}, \ldots, p_{0}^{N}\right)$ are initial conditions of a classical trajectory at time zero. Vectors $\boldsymbol{q}_{t}=\left(q_{t}^{1}, \ldots, q_{t}^{N}\right)$ and $\boldsymbol{p}_{t}=\left(p_{t}^{1}, \ldots, p_{t}^{N}\right)$ are its coordinates and momenta at time $t$. The classical action is

$$
S_{p q t}=\int_{0}^{t}\left[\boldsymbol{p}_{t^{\prime}} \cdot \dot{\boldsymbol{q}}_{t^{\prime}}-H\left(\boldsymbol{p}_{t^{\prime}}, \boldsymbol{q}_{t^{\prime}}, t^{\prime}\right)\right] d t^{\prime} .
$$

The prefactor carrying a trajectory stability information is

$$
R_{p q t}=\sqrt{\operatorname{det}(\boldsymbol{B})},
$$

with the matrix elements $\boldsymbol{B}=\left\{b_{i j}\right\}$ being

$$
b_{i j}=\frac{1}{2}\left(\frac{\partial p_{t}^{i}}{\partial p_{0}^{j}}+\frac{\partial q_{t}^{i}}{\partial q_{0}^{j}}-\frac{l \gamma}{\hbar} \frac{\partial q_{t}^{i}}{\partial p_{0}^{j}}+\frac{l}{\hbar \gamma} \frac{\partial p_{t}^{i}}{\partial q_{0}^{j}}\right) .
$$

The sign of the square root in Eq. (47) has to be chosen such that $R_{p q t}$ is a continuous function of time. ${ }^{34}$ The integration goes over all initial values $\left(\boldsymbol{q}_{0}, \boldsymbol{p}_{0}\right)$. The propagator is unitary in the stationary phase approximation, and it is time reversible. $^{35}$

The reactant and product wave packets, Eqs. (41) and (42), were setup as for the quantum-mechanical calculation, with the parameters $\alpha=4.5, q_{0}=4.7$, and $p_{0}=-7.0$. The overlap integrals of $g_{\gamma}\left(\boldsymbol{q}_{t}, \boldsymbol{p}_{t}, \boldsymbol{x}\right)$ with the reactant and product wave packets, needed to calculate the time-correlation functions, take the simple analytical form with this choice of the internal functions. The Wall-Porter potential was used for the semiclassical calculation as in Ref. 20. The reactant wave packets were propagated up to time $T=2760$ a.u. with the time step $d t=4.6$ a.u., and their time-dependent overlaps with the stationary product wave packets were Fourier transformed. The reactant-reactant correlation functions of time duration 918 a.u. were used to obtain the appropriate normalization in energy. The semiclassical normalization was identical to the exact normalization for practical purposes.

The cumulative reaction probability for the HK and QM propagation of the harmonic eigenstate wave packets is plotted in Fig. (5). The width of the expansion Gaussians was $\gamma / 2=\alpha=4.5$ a.u. in both dimensions. The sampling of trajectories was Gaussian-weighted Monter Carlo sampling. A single set of the classical trajectories contributed to all correlation functions. That made the timing of the exact quantum-mechanical and semiclassical calculations comparable. Here we used $10^{6}$ classical trajectories, but using 10 times fewer trajectories also gave semiquantitative agreement, although the discrepancies around the resonant and threshold energies were more pronounced. The matrix inversion in Eq. (31) did not pose a problem, since the reactantreactant correlation functions, which were nonzero for the first half of the propagation time, were obtained quite accurately.

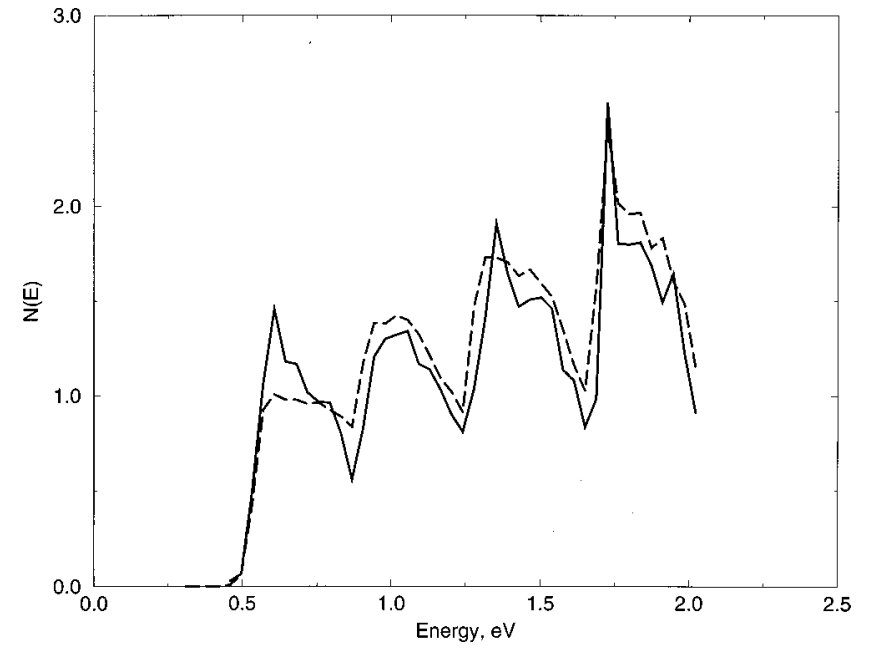

FIG. 5. The cumulative reaction probability, obtained from the semiclassical propagation of wave packets (solid line) and the quantum-mechanical result (dash line). The wave packets were initially located at $R_{0}=4.7$ a.u. in the translational coordinate and were constructed as the harmonic oscillator eigenstates in vibrational coordinate.

The presence of resonances in the $\mathrm{H}+\mathrm{H}_{2}$ system and the constricted geometry make the state-to-state reaction probabilities quite structured and challenging to describe semiclassically. The direct calculation of the semiclassical $N(E)$ performed here compares better with the quantummechanical results than the semiclassical calculation of individual $S$-matrix elements obtained previously. ${ }^{21}$ Moreover, the direct calculation of $N(E)$ is easier than obtaining all of the state-to-state probabilities, since no asymptotic states were required: The analytical form of the overlap integrals $\left\langle g_{\gamma}(0) \mid \Phi_{r}^{n}\right\rangle$ and $\left\langle\Phi_{r}^{n} \mid g_{\gamma}(t)\right\rangle$ between the HK Gaussians and the harmonic oscillator eigenstates made the calculation several times faster. In general, one also expects to find a reduction in the length of time that the trajectories need to be propagated relative to the state-to-state calculation, since it is not necessary for the trajectories to reach the asymptotic region. However, for the collinear hydrogen exchange reaction we observed no significant reduction in propagation time. This can be understood by recognizing that the resonances, which are the most challenging part of the calculation, are determined by trapped trajectories which spend far more time in the interaction region than in the approach or exit from this region.

\section{CONCLUSIONS}

We presented two new expressions, Eqs. (31) and (38), for the cumulative reaction probability using the trace expression $N(E)=\operatorname{Tr}\left(\boldsymbol{S}_{p r}^{\dagger}(E) \boldsymbol{S}_{p r}(E)\right)$. The expressions are cast in terms of the cross-correlation functions of wave packets which are incoming (outgoing) in translation and arbitrary in the internal degrees of freedom. This allows one to choose a convenient internal basis set, and to setup the reactant (product) wave packets closer to the interaction region, thus reducing the size of the numerical grid and the propagation time. Our formulation is explicitly symmetric with respect to reactants and products, i.e., both reactant and product wave packets can be propagated in time, forward and 
backward, respectively. The second formulation, which uses projection onto products, might be useful if the number of the product internal states is significantly larger than the number of the reactant internal states, despite the fact that an absorbing potential cannot be used as efficiently as in the symmetric formulation.

Both new formulations can be combined with the high resolution spectral method of filter-diagonalization to shorten the length of the propagation time. However, in our experience filter diagonalization works well only when used with an extremely accurate propagation method, such as Chebyshev method.

It would be very instructive to apply the present formulations to a system with a long range attractive potential. Here one might see a particularly large savings of the trace expression, Eq. (31) over a state-to-state calculation, by using convenient internal functions and setting up wave packets in the interaction region.

Perhaps most significant about the present formulation is that it can be readily combined with both exact and approximate time propagation methods. Previously, semiclassical calculations of $N(E)$ have been possible only in one dimension (1D); the present semiclassical calculation of $N(E)$ for collinear $\mathrm{H}+\mathrm{H}_{2}$ is a significant achievement, and shows the usefulness of the present formulation. We see no reason, in principle, that the present formulation implemented semiclassically cannot be applied to larger systems of greater chemical interest. As a first step in this direction, 3D calculations on $\mathrm{H}+\mathrm{H}_{2}$ are in progress. Finally, the formulation is also completely compatible with the time-dependent selfconsistent field (TDSCF) approach. In particular, by using an arbitrary internal basis it may be possible to reduce correlation between translation and internal degrees of freedom, increasing the accuracy and efficiency of TDSCF for reactive scattering.

${ }^{1}$ J. Taylor, Scattering Theory: The Quantum Theory of Nonrelativistic Collisions (Wiley, New York, 1972).
${ }^{2}$ R. G. Newton, Scattering Theory of Waves and Particles (SpringerVerlag, New York, 1982)

${ }^{3}$ W. H. Miller, S. D. Schwartz, and J. W. Tromp, J. Chem. Phys. 79, 4889 (1983).

${ }^{4}$ U. Manthe and W. H. Miller, J. Chem. Phys. 99, 3411 (1993).

${ }^{5}$ U. Manthe, T. Seideman, and W. H. Miller, J. Chem. Phys. 99, 10078 (1993).

${ }^{6}$ U. Manthe, T. Seideman, and W. H. Miller, J. Chem. Phys. 101, 4759 (1994).

${ }^{7}$ D. H. Zhang and J. C. Light, J. Chem. Phys. 104, 6184 (1996).

${ }^{8}$ D. H. Zhang and J. C. Light, J. Chem. Phys. 104, 4544 (1996).

${ }^{9}$ D. H. Zhang and J. C. Light, J. Chem. Phys. 106, 551 (1997).

${ }^{10}$ J. C. Light and D. H. Zhang, Faraday Discuss. (in press).

${ }^{11}$ S. M. Miller and T. C. Jr., Chem. Phys. Lett. 267, 417 (1997).

${ }^{12}$ D. J. Tannor and D. E. Weeks, J. Chem. Phys. 98, 3884 (1993).

${ }^{13}$ D. E. Weeks and D. J. Tannor, Chem. Phys. Lett. 207, 301 (1993).

${ }^{14}$ D. E. Weeks and D. J. Tannor, Chem. Phys. Lett. 224, 451 (1994).

${ }^{15}$ J. Q. Dai and J. Z. H. Zhang, J. Phys. Chem. 100, 6898 (1996).

${ }^{16}$ D. E. Manolopoulos, in Encyclopedia of Computational Chemistry, edited by P. v. R. Schleyer (Wiley, Chichester, 1998), Vol. 4.

${ }^{17}$ G. G. Balint-Kurti, R. N. Dixon, and C. C. Marston, J. Chem. Soc., Faraday Trans. 86, 1741 (1990).

${ }^{18}$ G. G. Balint-Kurti, R. N. Dixon, C. C. Marston, and A. J. Mulholland, Comput. Phys. Commun. 63, 126 (1991)

${ }^{19}$ F. Grossmann and E. Heller, Chem. Phys. Lett. 241, 45 (1995).

${ }^{20}$ S. Garashchuk and D. J. Tannor, Chem. Phys. Lett. 262, 477 (1996).

${ }^{21}$ S. Garashchuk, F. Grossmann, and D. J. Tannor, J. Chem. Soc., Faraday Trans. 93, 781 (1997).

${ }^{22}$ A. Jäckle and H.-D. Meyer, J. Chem. Phys. 105, 6778 (1996).

${ }^{23}$ B. W. Spath and W. Miller, Chem. Phys. Lett. 262, 486 (1996).

${ }^{24}$ S. Garashchuk and D. J. Tannor, J. Chem. Phys. 109, 3028 (1998).

${ }^{25}$ C. L. Russell, J. F. Castillo, D. E. Manolopoulos, and G. G. Balint-Kurti (unpublished).

${ }^{26}$ P. Siegban and B. Liu, J. Chem. Phys. 68, 2457 (1978).

${ }^{27}$ D. G. Truhlar and C. J. Horowitz, J. Chem. Phys. 68, 2466 (1978).

${ }^{28}$ W. Kolos and L. Wolniewicz, J. Chem. Phys. 43, 2429 (1965).

${ }^{29}$ M. D. Feit, J. A. Fleck, and A. Steiger, J. Comp. Physiol. 47, 412 (1982).

${ }^{30}$ M. R. Wall and D. Neuhauser, J. Chem. Phys. 102, 8011 (1995).

${ }^{31}$ V. A. Mandelshtam and H. S. Taylor, J. Chem. Phys. 102, 7390 (1995).

${ }^{32}$ V. A. Mandelshtam, in Multiparticle Quantum Scattering with Applications to Nuclear, Atomic and Molecular Physics, Vol. 89 of IMA Volumes in Mathematics and its Applications, edited by D. G. Truhlar and B. Simon (Springer, New York, 1997).

${ }^{33}$ M. Herman and E. Kluk, Chem. Phys. 91, 27 (1984).

${ }^{34}$ K. G. Kay, J. Chem. Phys. 100, 4377 (1994).

${ }^{35}$ M. Herman, J. Chem. Phys. 85, 2069 (1986). 